\title{
Robust Energy Management for Microgrids With High-Penetration Renewables
}

\author{
Yu Zhang, Student Member, IEEE, Nikolaos Gatsis, Member, IEEE, and Georgios B. Giannakis, Fellow, IEEE
}

\begin{abstract}
Due to its reduced communication overhead and robustness to failures, distributed energy management is of paramount importance in smart grids, especially in microgrids, which feature distributed generation (DG) and distributed storage (DS). Distributed economic dispatch for a microgrid with high renewable energy penetration and demand-side management operating in grid-connected mode is considered in this paper. To address the intrinsically stochastic availability of renewable energy sources (RES), a novel power scheduling approach is introduced. The approach involves the actual renewable energy as well as the energy traded with the main grid, so that the supplydemand balance is maintained. The optimal scheduling strategy minimizes the microgrid net cost, which includes DG and DS costs, utility of dispatchable loads, and worst-case transaction cost stemming from the uncertainty in RES. Leveraging the dual decomposition, the optimization problem formulated is solved in a distributed fashion by the local controllers of DG, DS, and dispatchable loads. Numerical results are reported to corroborate the effectiveness of the novel approach.
\end{abstract}

Index Terms-Demand side management, distributed algorithms, distributed energy resources, economic dispatch, energy management, microgrids, renewable energy, robust optimization.

\section{NOMENCLATURE}

A. Indices, numbers, and sets

$T, t \quad$ Number of scheduling periods, period index.

$M, m$ Number of conventional distributed generation (DG) units, and their index.

$N, n \quad$ Number of dispatchable (class-1) loads, load index.

$Q, q \quad$ Number of energy (class-2) loads, load index.

$J, j \quad$ Number of distributed storage (DS) units, and their index.

$I, i \quad$ Number of power production facilities with renewable energy source (RES), and facility index.

$S, s \quad$ Number of sub-horizons, and sub-horizon index.

$k \quad$ Algorithm iteration index.

$\mathcal{T} \quad$ Set of time periods in the scheduling horizon.

$\mathcal{T}_{s} \quad$ Sub-horizon $s$ for all RES facilities.

$\mathcal{T}_{i, s} \quad$ Sub-horizon $s$ for RES facility $i$.

$\mathcal{M}$ Set of conventional DG units.

$\mathcal{N} \quad$ Set of dispatchable loads.

$\mathcal{Q} \quad$ Set of energy loads.

This work was supported by the University of Minnesota Institute of Renewable Energy and the Environment (IREE) under Grant RL-0010-13. This work was presented in part at the 3rd IEEE Intl. Conf. on Smart Grid Commun., Tainan, Taiwan, November 5-8, 2012.

The authors are with the Dept. of ECE and the Digital Technology Center, University of Minnesota, Minneapolis, MN 55455, USA. Tel/fax: +1(612)6249510/625-2002. E-mails: \{zhan1220, gatsisn, georgios\}@umn.edu
$\mathcal{J} \quad$ Set of DS units.

$\mathcal{W} \quad$ Power output uncertainty set for all RES facilities.

$\mathcal{W}_{i} \quad$ Power output uncertainty set of RES facility $i$.

\section{B. Constants}

$P_{G_{m}}^{\min }, P_{G_{m}}^{\max } \quad$ Minimum and maximum power output of conventional DG unit $m$.

$R_{m \text {,up }}, R_{m \text {,down }}$ Ramp-up and ramp-down limits of conventional DG unit $m$.

$\mathrm{SR}^{t} \quad$ Spinning reserve for conventional DG.

$L^{t} \quad$ Fixed power demand of critical loads in period $t$.

$P_{D_{n}}^{\min }, P_{D_{n}}^{\max } \quad$ Minimum and maximum power consump-

$P_{E_{q}}^{\min , t}, P_{E_{q}}^{\max , t} \quad$ Minimum and maximum power consumption of load $q$ in period $t$.

$S_{q}, T_{q} \quad$ Power consumption start and stop times of load $q$.

$E_{q}^{\max } \quad$ Total energy consumption of load $q$ from start time $S_{q}$ to termination time $T_{q}$.

$P_{B_{j}}^{\min }, P_{B_{j}}^{\max } \quad$ Minimum and maximum (dis)charging power of DS unit $j$.

$B_{j}^{\min }$

$B_{j}^{\max }$

$\eta_{j}$

$P_{R}^{\min }, P_{R}^{\max }$

$\underline{W}_{i}^{t}, \bar{W}_{i}^{t}$

$W_{i, s}^{\min }, W_{i, s}^{\max }$

Minimum stored energy of DS unit $j$ in period $T$.

Capacity of DS unit $j$.

Efficiency of DS unit $j$.

Lower and upper bounds for $P_{R}^{t}$.

Minimum and maximum forecasted power output of RES facility $i$ in $t$.

Minimum and maximum forecasted total wind power of wind farm $i$ across subhorizon $\mathcal{T}_{i, s}$.

$W_{s}^{\min }, W_{s}^{\max } \quad$ Minimum and maximum forecasted total wind power of all wind farms across subhorizon $\mathcal{T}_{s}$.

$\alpha^{t}, \beta^{t} ; \gamma^{t}, \delta^{t} \quad$ Purchase and selling prices; and functions thereof.

$\pi_{q}^{t} \quad$ Parameter of utility function of load $q$.

$\mathrm{DOD}_{j} ; \psi_{j}^{t} \quad$ Depth of discharge specification of DS unit $j$; and parameters of storage cost.

\section{Uncertain quantities}

$W_{i}^{t} \quad$ Power output from RES facility $i$ in period $t$.

D. Decision variables

$P_{G_{m}}^{t} \quad$ Power output of DG unit $m$ in period $t$. 
$P_{D_{n}}^{t} \quad$ Power consumption of load $n$ in period $t$.

$P_{E_{q}}^{t}$

$P_{B_{j}}^{t}$

$B_{j}^{t}$

$P_{R}^{t}$

$\tilde{P}_{R}^{t}$

$\mathbf{X}$

$\lambda^{t}, \mu^{t}, \nu^{t}$

$\mathbf{z}$

$W_{\text {worst }}^{t}$

\section{E. Functions}

$C_{m}^{t}(\cdot)$

$U_{D_{n}}^{t}(\cdot)$

$U_{E_{q}}^{t^{n}}(\cdot)$

$H_{j}^{t}(\cdot)$

$G(\cdot, \cdot)$

$G(\cdot), \tilde{G}(\cdot)$

$\mathcal{L}(\mathbf{x}, \mathbf{z})$

$\mathcal{D}(\mathbf{z})$

Power consumption of load $q$ in period $t$. $t$. period $t$.

the RES and storage in period $t$.

Auxiliary variable.

Vector collecting all decision variables.

Lagrange multipliers.

Vector collecting all Lagrange multipliers. yielding the worst-case transaction cost.

Utility of load $n$ in period $t$.

Utility of load $q$ in period $t$.

Cost of DS unit $j$ in period $t$.

Modified worst-case transaction cost.

Lagrangian function.

Dual function.
(Dis)charging power of DS unit $j$ in period

Stored energy of DS unit $j$ at the end of the

Net power delivered to the microgrid from

Power production from all RES facilities in $t$

Cost of conventional DG unit $m$ in period $t$.

Worst transaction cost across entire horizon.

\section{INTRODUCTION}

Microgrids are power systems comprising distributed energy resources (DERs) and electricity end-users, possibly with controllable elastic loads, all deployed across a limited geographic area [1]. Depending on their origin, DERs can come either from distributed generation (DG) or from distributed storage (DS). DG refers to small-scale power generators such as diesel generators, fuel cells, and renewable energy sources (RES), as in wind or photovoltaic (PV) generation. DS paradigms include batteries, flywheels, and pumped storage. Specifically, DG brings power closer to the point it is consumed, thereby incurring fewer thermal losses and bypassing limitations imposed by a congested transmission network. Moreover, the increasing tendency towards high penetration of RES stems from their environment-friendly and price-competitive advantages over conventional generation. Typical microgrid loads include critical non-dispatchable types and elastic controllable ones.

Microgrids operate in grid-connected or island mode, and may entail distribution networks with residential or commercial end-users, in rural or urban areas. A typical configuration is depicted in Fig. 1] see also [1]. The microgrid energy manager (MGEM) coordinates the DERs and the controllable loads. Each of the DERs and loads has a local controller (LC), which coordinates with the MGEM the scheduling of resources through the communications infrastructure in a distributed fashion. The main challenge in energy scheduling is to account for the random and nondispatchable nature of the RES.

Optimal energy management for microgrids including economic dispatch (ED), unit commitment (UC), and demandside management (DSM) is addressed in [2], but without pursuing a robust formulation against RES uncertainty. Based

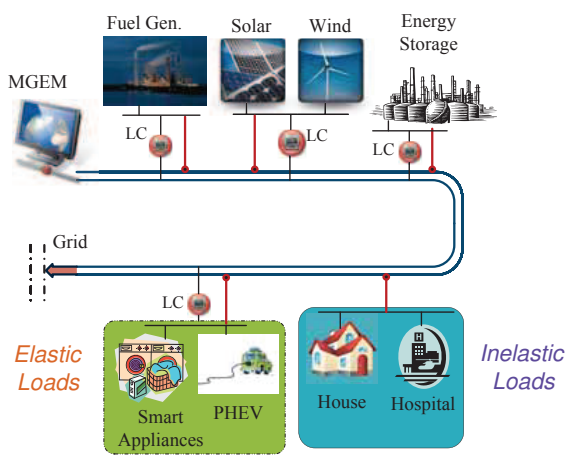

Fig. 1. Distributed control and computation architecture of a microgrid.

on the Weibull distribution for wind speed and the wind-speedto-power-output mappings, an ED problem is formulated to minimize the risk of overestimation and underestimation of available wind power [3]. Stochastic programming is also used to cope with the variability of RES. Single-period chanceconstrained ED problems for RES have been studied in [4], yielding probabilistic guarantees that the load will be served. Considering the uncertainties of demand profiles and PV generation, a stochastic program is formulated to minimize the overall cost of electricity and natural gas for a building in [5]. Without DSM, robust scheduling problems with penalty-based costs for uncertain supply and demand have been investigated in [6]. Recent works explore energy scheduling with DSM and RES using only centralized algorithms [7], [8]. An energy source control and DS planning problem for a microgrid is formulated and solved using model predictive control in [9]. Distributed algorithms are developed in [10], but they only coordinate DERs to supply a given load without considering the stochastic nature of RES. Recently, a worst-case transaction cost based energy scheduling scheme has been proposed to address the variability of RESs through robust optimization that can also afford distributed implementation [11]. However, [11] considers only a single wind farm and no DS, and its approach cannot be readily extended to include multiple RESs and DS.

The present paper deals with optimal energy management for both supply and demand of a grid-connected microgrid incorporating RES. The objective of minimizing the microgrid net cost accounts for conventional DG cost, utility of elastic loads, penalized cost of DS, and a worst-case transaction cost. The latter stems from the ability of the microgrid to sell excess energy to the main grid, or to import energy in case of shortage. A robust formulation accounting for the worstcase amount of harvested RES is developed. A novel model is introduced in order to maintain the supply-demand balance arising from the intermittent RES. Moreover, a transactionprice-based condition is established to ensure convexity of the overall problem (Section [II). The separable structure and strong duality of the resultant problem are leveraged to develop a low-overhead distributed algorithm based on dual decomposition, which is computationally efficient and resilient to communication outages or attacks. For faster convergence, the proximal bundle method is employed for the non-smooth subproblem handled by the LC of RES (Section IIII). Numerical results corroborate the merits of the novel designs (Section IV], and the paper is wrapped up with a concluding 
summary (Section $\nabla$.

Compared to [11], the contribution of the paper is threefold, and of critical importance for microgrids with high-penetration renewables. First, a detailed model for DS is included, and different design choices for storage cost functions are given to accommodate, for example, depth-of-discharge specifications. Second, with the envisioned tide of high-penetration renewable energy, multiple wind farms are considered alongside two pertinent uncertainty models. Finally, a new class of controllable loads is added, with each load having a requirement of total energy over the scheduling horizon, as is the case with charging of plug-in hybrid electric vehicles (PHEVs). Detailed numerical tests are presented to illustrate the merits of the scheduling decisions for the DG, DS, and controllable loads. Notation. Boldface lower case letters represent vectors; $\mathbb{R}^{n}$ and $\mathbb{R}$ stand for spaces of $n \times 1$ vectors and real numbers, respectively; $\mathbb{R}_{+}^{n}$ is the $n$-dimensional non-negative orthant; $\mathbf{x}^{\prime}$ transpose, and $\|\mathbf{x}\|$ the Euclidean norm of $\mathbf{x}$.

\section{Robust Energy Management Formulation}

Consider a microgrid comprising $M$ conventional (fossil fuel) generators, $I$ RES facilities, and $J$ DS units (see also Fig. 11. The scheduling horizon is $\mathcal{T}:=\{1,2, \ldots, T\}$ (e.g., one-day ahead). The particulars of the optimal scheduling problem are explained in the next subsections.

\section{A. Load Demand Model}

Loads are classified in two categories. The first comprises inelastic loads, whose power demand should be satisfied at all times. Examples are power requirements of hospitals or illumination demand from residential areas.

The second category consists of elastic loads, which are dispatchable, in the sense that their power consumption is adjustable, and can be scheduled. These loads can be further divided in two classes, each having the following characteristics:

i) The first class contains loads with power consumption $P_{D_{n}}^{t} \in\left[P_{D_{n}}^{\min }, P_{D_{n}}^{\max }\right]$, where $n \in \mathcal{N}:=\{1, \ldots, N\}$, and $t \in \mathcal{T}$. Higher power consumption yields higher utility for the end user. The utility function of the $n$th dispatchable load, $U_{D_{n}}^{t}\left(P_{D_{n}}^{t}\right)$, is selected to be increasing and concave, with typical choices being piecewise linear or smooth quadratic; see also [12]. An example from this class is an $\mathrm{A} / \mathrm{C}$.

ii) The second class includes loads indexed by $q \in \mathcal{Q}:=$ $\{1, \ldots, Q\}$ with power consumption limits $P_{E_{q}}^{\min }$ and $P_{E_{q}}^{\max }$, and prescribed total energy requirements $E_{q}$ which have to be achieved from the start time $S_{q}$ to termination time $T_{q}$; see e.g., [13]. This type of loads can be the plug-in hybrid electric vehicles (PHEVs). Power demand variables $\left\{P_{E_{q}}^{t}\right\}_{t=1}^{T}$ therefore are constrained as $\sum_{t=S_{q}}^{T_{q}} P_{E_{q}}^{t}=E_{q}$ and $P_{E_{q}}^{t} \in\left[P_{E_{q}}^{\min , t}, P_{E_{q}}^{\max , t}\right], t \in \mathcal{T}$, while $P_{E_{q}}^{\min , t}=P_{E_{q}}^{\max , t}=0$ for $t \notin\left\{S_{q}, \ldots, T_{q}\right\}$. Higher power consumption in earlier slots as opposed to later slots may be desirable for a certain load, so that the associated task finishes earlier. This behavior can be encouraged by adopting for the $q$ th load an appropriately designed time-varying concave utility function $U_{E_{q}}^{t}\left(P_{E_{q}}^{t}\right)$. An example is $U_{E_{q}}^{t}\left(P_{E_{q}}^{t}\right):=\pi_{q}^{t} P_{E_{q}}^{t}$, with weights $\left\{\pi_{q}^{t}\right\}$ decreasing in $t$ from slots $S_{q}$ to $T_{q}$. Naturally, $U_{E_{q}}^{t}\left(P_{E_{q}}^{t}\right) \equiv 0$ can be selected if the consumer is indifferent to how power is consumed across slots.

\section{B. Distributed Storage Model}

Let $B_{j}^{t}$ denote the stored energy of the $j$ th battery at the end of the slot $t$, with initial available energy $B_{j}^{0}$ while $B_{j}^{\max }$ denotes the battery capacity, so that $0 \leq B_{j}^{t} \leq B_{j}^{\max }, j \in$ $\mathcal{J}:=\{1, \ldots, J\}$. Let $P_{B_{j}}^{t}$ be the power delivered to (drawn from) the $j$ th storage device at slot $t$, which amounts to charging $\left(P_{B_{j}}^{t} \geq 0\right)$ or discharging $\left(P_{B_{j}}^{t} \leq 0\right)$ of the battery. Clearly, the stored energy obeys the dynamic equation

$$
B_{j}^{t}=B_{j}^{t-1}+P_{B_{j}}^{t}, j \in \mathcal{J}, t \in \mathcal{T} .
$$

Variables $P_{B_{j}}^{t}$ are constrained in the following ways:

i) The amount of (dis)charging is bounded, that is

$$
\begin{aligned}
P_{B_{j}}^{\min } & \leq P_{B_{j}}^{t} \leq P_{B_{j}}^{\max } \\
-\eta_{j} B_{j}^{t-1} & \leq P_{B_{j}}^{t}
\end{aligned}
$$

with bounds $P_{B_{j}}^{\min }<0$ and $P_{B_{j}}^{\max }>0$, while $\eta_{j} \in(0,1]$ is the efficiency of DS unit $j$ [14], [15]. The constraint in (3) means that a fraction $\eta_{j}$ of the stored energy $B_{j}^{t-1}$ is available for discharge.

ii) Final stored energy is also bounded for the sake of future scheduling horizons, that is $B_{j}^{T} \geq B_{j}^{\mathrm{min}}$.

To maximize DS lifetime, a storage cost $H_{j}^{t}\left(B_{j}^{t}\right)$ can be employed to encourage the stored energy to remain above a specified depth of discharge, denoted as $\mathrm{DOD}_{j} \in[0,1]$, where $100 \%(0 \%)$ depth of discharge means the battery is empty (full) [15]. Such a cost is defined as $H_{j}^{t}\left(B_{j}^{t}\right):=\psi_{j}^{t}[(1-$ $\left.\left.\mathrm{DOD}_{j}\right) B_{j}^{\max }-B_{j}^{t}\right]$. Note that the storage cost $H_{j}^{t}\left(B_{j}^{t}\right)$ can be interpreted as imposing a soft constraint preventing large variations of the stored energy. Clearly, higher weights $\left\{\psi_{j}^{t}\right\}$ encourage smaller variation. If high power exchange is to be allowed, these weights can be chosen very small, or one can even select $H_{j}^{t}\left(B_{j}^{t}\right) \equiv 0$ altogether.

\section{Worst-case Transaction Cost}

Let $W_{i}^{t}$ denote the actual renewable energy harvested by the $i$ th RES facility at time slot $t$, and also let $\mathbf{w}$ collect all $W_{i}^{t}$, i.e., $\mathbf{w}:=\left[W_{1}^{1}, \ldots, W_{1}^{T}, \ldots, W_{I}^{1}, \ldots, W_{I}^{T}\right]$. To capture the intrinsically stochastic and time-varying availability of RES, it is postulated that $\mathbf{w}$ is unknown, but lies in a polyhedral uncertainty set $\mathcal{W}$. The following are two practical examples.

i) The first example postulates a separate uncertainty set $\mathcal{W}_{i}$ for each RES facility in the form

$$
\begin{aligned}
& \mathcal{W}_{i}:=\left\{\left\{W_{i}^{t}\right\}_{t=1}^{T} \mid \underline{W}_{i}^{t} \leq W_{i}^{t} \leq \bar{W}_{i}^{t},\right. \\
& \left.W_{i, s}^{\min } \leq \sum_{t \in \mathcal{T}_{i, s}} W_{i}^{t} \leq W_{i, s}^{\max }, \mathcal{T}=\bigcup_{s=1}^{S} \mathcal{T}_{i, s}\right\}
\end{aligned}
$$


where $\underline{W}_{i}^{t}\left(\bar{W}_{i}^{t}\right)$ denotes a lower (upper) bound on $W_{i}^{t} ; \mathcal{T}$ is partitioned into consecutive but non-overlapping subhorizons $\mathcal{T}_{i, s}$ for $i=1, \ldots, I, s=1,2, \ldots, S$; the total renewable energy for the $i$ th RES facility over the $s$ th sub-horizon is assumed bounded by $W_{i, s}^{\min }$ and $W_{i, s}^{\max }$. In this example, $\mathcal{W}$ takes the form of Cartesian product

$$
\mathcal{W}=\mathcal{W}_{1} \times \ldots \times \mathcal{W}_{I}
$$

ii) The second example assumes a joint uncertainty model across all the RES facilities as

$$
\begin{aligned}
& \mathcal{W}:=\left\{\mathbf{w} \mid \underline{W}_{i}^{t} \leq W_{i}^{t} \leq \bar{W}_{i}^{t},\right. \\
& \left.W_{s}^{\min } \leq \sum_{t \in \mathcal{T}_{s}} \sum_{i=1}^{I} W_{i}^{t} \leq W_{s}^{\max }, \mathcal{T}=\bigcup_{s=1}^{S} \mathcal{T}_{s}\right\}
\end{aligned}
$$

where $\underline{W}_{i}^{t}\left(\bar{W}_{i}^{t}\right)$ denotes a lower (upper) bound on $W_{i}^{t}$; $\mathcal{T}$ is partitioned into consecutive but non-overlapping sub-horizons $\mathcal{T}_{s}$ for $s=1,2, \ldots, S$; the total renewable energy harvested by all the RES facilities over the $s$ th sub-horizon is bounded by $W_{s}^{\min }$ and $W_{s}^{\max }$; see also [8].

The previous two RES uncertainty models are quite general and can take into account different geographical and meteorological factors. The only information required is the deterministic lower and upper bounds, namely $\underline{W}_{i}^{t}, \bar{W}_{i}^{t}, W_{i, s}^{\min }$, $W_{i, s}^{\max }, W_{s}^{\min }, W_{s}^{\max }$, which can be determined via inference schemes based on historical data [16].

Supposing the microgrid operates in a grid-connected mode, a transaction mechanism between the microgrid and the main grid is present, whereby the microgrid can buy/sell energy from/to the spot market. Let $P_{R}^{t}$ be an auxiliary variable denoting the net power delivered to the microgrid from the renewable energy sources and the distributed storage in order to maintain the supply-demand balance at slot $t$. The shortage energy per slot $t$ is given by $\left[P_{R}^{t}-\sum_{i=1}^{I} W_{i}^{t}+\sum_{j=1}^{J} P_{B_{j}}^{t}\right]^{+}$, while the surplus energy is $\left[P_{R}^{t}-\sum_{i=1}^{I} W_{i}^{t}+\sum_{j=1}^{J} P_{B_{j}}^{t}\right]^{-}$, where $[a]^{+}:=\max \{a, 0\}$, and $[a]^{-}:=\max \{-a, 0\}$.

The amount of shortage energy is bought with known purchase price $\alpha^{t}$, while the surplus energy is sold to the main grid with known selling price $\beta^{t}$. The worst-case net transaction cost is thus given by

$$
\begin{aligned}
G\left(\left\{P_{R}^{t}\right\},\left\{P_{B_{j}}^{t}\right\}\right) & :=\max _{\mathbf{w} \in \mathcal{W}} \sum_{t=1}^{T}\left(\alpha^{t}\left[P_{R}^{t}-\sum_{i=1}^{I} W_{i}^{t}+\sum_{j=1}^{J} P_{B_{j}}^{t}\right]^{+}\right. \\
& \left.-\beta^{t}\left[P_{R}^{t}-\sum_{i=1}^{I} W_{i}^{t}+\sum_{j=1}^{J} P_{B_{j}}^{t}\right]^{-}\right)
\end{aligned}
$$

where $\left\{P_{R}^{t}\right\}$ collects $P_{R}^{t}$ for $t=1,2, \ldots, T$ and $\left\{P_{B_{j}}^{t}\right\}$ collects $P_{B_{j}}^{t}$ for $j=1,2, \ldots, J, t=1,2, \ldots, T$.

Remark 1. (Worst-case model versus stochastic model). The worst-case robust model advocated here is particularly attractive when the probability distribution of the renewable power production is unavailable. This is for instance the case for multiple wind farms, where the spatio-temporal joint distribution of the wind power generation is intractable (see detailed discussions in [17] and [18]). If an accurate probabilistic model is available, an expectation-based stochastic program can be formulated to bypass the conservatism of worst-case optimization. In the case of wind generation, suppose that wind power $W_{i}^{t}$ is a function of the random wind velocity $v_{i}^{t}$, for which different models are available, and the wind-speed-to-poweroutput mappings $W_{i}^{t}\left(v_{i}^{t}\right)$ are known [19]. Then, the worst-case transaction cost can be replaced by the expected transaction $\operatorname{cost} G\left(\left\{P_{R}^{t}\right\},\left\{P_{B_{j}}^{t}\right\}\right):=\mathbb{E}_{\mathbf{v}}\left(\sum_{t=1}^{T} \alpha^{t}\left[P_{R}^{t}-\sum_{i=1}^{I} W_{i}^{t}\left(v_{i}^{t}\right)+\right.\right.$ $\left.\left.\sum_{j=1}^{J} P_{B_{j}}^{t}\right]^{+}-\beta^{t}\left[P_{R}^{t}-\sum_{i=1}^{I} W_{i}^{t}\left(v_{i}^{t}\right)+\sum_{j=1}^{J} P_{B_{j}}^{t}\right]^{-}\right)$, where $\mathbf{v}$ collects $v_{i}^{t}$ for all $i$ and $t$.

\section{Microgrid Energy Management Problem}

Apart from RES, microgrids typically entail also conventional DG. Let $P_{G_{m}}^{t}$ be the power produced by the $m$ th conventional generator, where $m \in \mathcal{M}:=\{1, \ldots, M\}$ and $t \in \mathcal{T}$. The cost of the $m$ th generator is given by an increasing convex function $C_{m}^{t}\left(P_{G_{m}}^{t}\right)$, which typically is either piecewise linear or smooth quadratic.

The energy management problem amounts to minimizing the microgrid social net cost; that is, the cost of conventional generation, storage, and the worst-case transaction cost (due to the volatility of RES) minus the utility of dispatchable loads:

$$
\begin{aligned}
& \text { (P1) } \min _{\substack{\left\{P_{G_{m}}^{t}, P_{D_{n}}^{t}, P_{E_{q}}^{t}, B_{j}^{t}, P_{B_{j}}^{t}, P_{R}^{t}\right\}}} \sum_{t=1}^{T}\left(\sum_{m=1}^{M} C_{m}^{t}\left(P_{G_{m}}^{t}\right)-\sum_{n=1}^{N} U_{D_{n}}^{t}\left(P_{D_{n}}^{t}\right)\right. \\
& \left.-\sum_{q=1}^{Q} U_{E_{q}}^{t}\left(P_{E_{q}}^{t}\right)+\sum_{j=1}^{J} H_{j}^{t}\left(B_{j}^{t}\right)\right)+G\left(\left\{P_{R}^{t}\right\},\left\{P_{B_{j}}^{t}\right\}\right)
\end{aligned}
$$

subject to:

$P_{G_{m}}^{\min } \leq P_{G_{m}}^{t} \leq P_{G_{m}}^{\max }, m \in \mathcal{M}, t \in \mathcal{T}$

$P_{G_{m}}^{t}-P_{G_{m}}^{t-1} \leq R_{m, \text { up }}, m \in \mathcal{M}, t \in \mathcal{T}$

$P_{G_{m}}^{t-1}-P_{G_{m}}^{t} \leq R_{m, \text { down }}, m \in \mathcal{M}, t \in \mathcal{T}$

$\sum_{m=1}^{M}\left(P_{G_{m}}^{\max }-P_{G_{m}}^{t}\right) \geq \mathrm{SR}^{t}, t \in \mathcal{T}$

$P_{D_{n}}^{\min } \leq P_{D_{n}}^{t} \leq P_{D_{n}}^{\max }, n \in \mathcal{N}, t \in \mathcal{T}$

$P_{E_{q}}^{\min , t} \leq P_{E_{q}}^{t} \leq P_{E_{q}}^{\max , t}, q \in \mathcal{Q}, t \in \mathcal{T}$

$\sum_{t=S_{q}}^{T_{q}} P_{E_{q}}^{t}=E_{q}, q \in \mathcal{Q}$

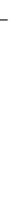

$$
\begin{aligned}
& 0 \leq B_{j}^{t} \leq B_{j}^{\max }, B_{j}^{T} \geq B_{j}^{\min }, j \in \mathcal{J}, t \in \mathcal{T} \\
& P_{B_{j}}^{\min } \leq P_{B_{j}}^{t} \leq P_{B_{j}}^{\max }, j \in \mathcal{J}, t \in \mathcal{T} \\
& -\eta_{j} B_{j}^{t-1} \leq P_{B_{j}}^{t}, j \in \mathcal{J}, t \in \mathcal{T} \\
& B_{j}^{t}=B_{j}^{t-1}+P_{B_{j}}^{t}, j \in \mathcal{J}, t \in \mathcal{T} \\
& P_{R}^{\min } \leq P_{R}^{t} \leq P_{R}^{\max }, t \in \mathcal{T} \\
& \sum_{m=1}^{M} P_{G_{m}}^{t}+P_{R}^{t}=L^{t}+\sum_{n=1}^{N} P_{D_{n}}^{t}+\sum_{q=1}^{Q} P_{E_{q}}^{t}, t \in \mathcal{T} .
\end{aligned}
$$

Constraints 8b-8e stand for the minimum/maximum power output, ramping up/down limits, and spinning reserves, 
respectively, which capture the typical physical requirements of a power generation system. Constraints $8 \mathrm{8t}$ and $8 \mathrm{~m}$ correspond to the minimum/maximum power of the flexible load demand and committed renewable energy. Constraint $8 \mathrm{nn}$ is the power supply-demand balance equation ensuring the total demand is satisfied by the power generation at any time.

Note that constraints $8 \mathrm{~b}-8 \mathrm{n}$ are linear, while $C_{m}^{t}(\cdot)$, $-U_{D_{n}}^{t}(\cdot),-U_{E_{q}}^{t}(\cdot)$, and $H_{j}^{t}(\cdot)$ are convex (possibly nondifferentiable or non-strictly convex) functions. Consequently, the convexity of (P1) depends on that of $G\left(\left\{P_{R}^{t}\right\},\left\{P_{B_{j}}^{t}\right\}\right)$, which is established in the following proposition.

Proposition 1. If the selling price $\beta^{t}$ does not exceed the purchase price $\alpha^{t}$ for any $t \in \mathcal{T}$, then the worst-case transaction cost $G\left(\left\{P_{R}^{t}\right\},\left\{P_{B_{j}}^{t}\right\}\right)$ is convex in $\left\{P_{R}^{t}\right\}$ and $\left\{P_{B_{j}}^{t}\right\}$.

Proof: Using that $[a]^{+}+[a]^{-}=|a|$, and $[a]^{+}-[a]^{-}=a$, $G\left(\left\{P_{R}^{t}\right\},\left\{P_{B_{j}}^{t}\right\}\right)$ can be re-written as

$$
\begin{aligned}
G\left(\left\{P_{R}^{t}\right\},\left\{P_{B_{j}}^{t}\right\}\right) & =\max _{\mathbf{w} \in \mathcal{W}} \sum_{t=1}^{T}\left(\delta^{t}\left|P_{R}^{t}-\sum_{i=1}^{I} W_{i}^{t}+\sum_{j=1}^{J} P_{B_{j}}^{t}\right|\right. \\
+ & \left.\gamma^{t}\left(P_{R}^{t}-\sum_{i=1}^{I} W_{i}^{t}+\sum_{j=1}^{J} P_{B_{j}}^{t}\right)\right)
\end{aligned}
$$

with $\delta^{t}:=\left(\alpha^{t}-\beta^{t}\right) / 2$, and $\gamma^{t}:=\left(\alpha^{t}+\beta^{t}\right) / 2$. Since the absolute value function is convex, and the operations of nonnegative weighted summation and pointwise maximum (over an infinite set) preserve convexity [20, Sec. 3.2], the claim follows readily.

An immediate corollary of Proposition 1 is that the energy management problem (P1) is convex if $\beta^{t} \leq \alpha^{t}$ for all $t$. The next section focuses on this case, and designs an efficient decentralized solver for (P1).

\section{Distributed Algorithm}

In order to facilitate a distributed algorithm for (P1), a variable transformation is useful. Specifically, upon introducing $\tilde{P}_{R}^{t}:=P_{R}^{t}+\sum_{j=1}^{J} P_{B_{j}}^{t}$, (P1) can be re-written as

$$
\begin{aligned}
& \min _{\mathbf{x}} \sum_{t=1}^{T}\left(\sum_{m=1}^{M} C_{m}^{t}\left(P_{G_{m}}^{t}\right)-\sum_{n=1}^{N} U_{D_{n}}^{t}\left(P_{D_{n}}^{t}\right)\right. \\
& \left.-\sum_{q=1}^{Q} U_{E_{q}}^{t}\left(P_{E_{q}}^{t}\right)+\sum_{j=1}^{J} H_{j}^{t}\left(B_{j}^{t}\right)\right)+G\left(\left\{\tilde{P}_{R}^{t}\right\}\right) \\
& \text { subject to: } \quad \underline{8 \mathrm{~b}}-\underline{8 \mathrm{n}} \\
& \tilde{P}_{R}^{t}=P_{R}^{t}+\sum_{j=1}^{J} P_{B_{j}}^{t}, t \in \mathcal{T}
\end{aligned}
$$

where $\mathrm{x}$ collects all the primal variables $\left\{P_{G_{m}}^{t}, P_{D_{n}}^{t}, P_{E_{q}}^{t}, P_{B_{j}}^{t}, B_{j}^{t}, P_{R}^{t}, \tilde{P}_{R}^{t}\right\} ; \quad\left\{\tilde{P}_{R}^{t}\right\} \quad$ collects $\quad \tilde{P}_{R}^{t}$ for $t=1, \ldots, T$; and

$G\left(\left\{\tilde{P}_{R}^{t}\right\}\right):=\max _{\mathbf{w} \in \mathcal{W}} \sum_{t=1}^{T}\left(\delta^{t}\left|\tilde{P}_{R}^{t}-\sum_{i=1}^{I} W_{i}^{t}\right|+\gamma^{t}\left(\tilde{P}_{R}^{t}-\sum_{i=1}^{I} W_{i}^{t}\right)\right)$

The following proposition extends the result of Proposition 1 to the transformed problem, and asserts its strong duality.
Proposition 2. If $(P 2)$ is feasible, and the selling price $\beta^{t}$ does not exceed the purchase price $\alpha^{t}$ for any $t \in \mathcal{T}$, then there is no duality gap.

Proof: Due to the strong duality theorem for the optimization problems with linear constraints (cf. [21, Prop. 5.2.1]), it suffices to show that the cost function is convex over the entire space and its optimal value is finite. First, using the same argument, convexity of $G\left(\left\{\tilde{P}_{R}^{t}\right\}\right)$ in $\left\{\tilde{P}_{R}^{t}\right\}$ is immediate under the transaction price condition. The finiteness of the optimal value is guaranteed by the fact that the continuous convex cost 10a is minimized over a nonempty compact set specified by $(8 \mathrm{~b}-8 \mathrm{n}$ ), and $110 \mathrm{~b}$ ).

The strong duality asserted by Proposition 2 motivates the use of Lagrangian relaxation techniques in order to solve the scheduling problem. Moreover, problem (P2) is clearly separable, meaning that its cost and constraints are sums of terms, with each term dependent on different optimization variables. The features of strong duality and separability imply that Lagrangian relaxation and dual decomposition are applicable to yield a decentralized algorithm; see also related techniques in power systems [22] and communication networks [23], [24]. Coordinated by dual variables, the dual approach decomposes the original problem into several separate subproblems that can be solved by the LCs in parallel. The development of the distributed algorithm is undertaken next.

\section{A. Dual Decomposition}

Constraints $8 \mathrm{e}$, $8 \mathrm{n}$, and $10 \mathrm{~b}$ couple variables across generators, loads, and the RES. Let $\mathbf{z}$ collect dual variables $\left\{\mu^{t}\right\},\left\{\lambda^{t}\right\}$, and $\left\{\nu^{t}\right\}$, which denote the corresponding Lagrange multipliers. Keeping the remaining constraints implicit, the partial Lagrangian is given by

$$
\begin{aligned}
& \mathcal{L}(\mathbf{x}, \mathbf{z})=\sum_{t=1}^{T}\left(\sum_{m=1}^{M} C_{m}^{t}\left(P_{G_{m}}^{t}\right)-\sum_{n=1}^{N} U_{D_{n}}^{t}\left(P_{D_{n}}^{t}\right)\right. \\
& \left.-\sum_{q=1}^{Q} U_{E_{q}}^{t}\left(P_{E_{q}}^{t}\right)+\sum_{j=1}^{J} H_{j}^{t}\left(B_{j}^{t}\right)\right)+G\left(\left\{\tilde{P}_{R}^{t}\right\}\right) \\
& +\sum_{t=1}^{T}\left\{\mu^{t}\left(\mathrm{SR}^{t}-\sum_{m=1}^{M}\left(P_{G_{m}}^{\max }-P_{G_{m}}^{t}\right)\right)\right. \\
& -\lambda^{t}\left(\sum_{m=1}^{M} P_{G_{m}}^{t}+P_{R}^{t}-\sum_{n=1}^{N} P_{D_{n}}^{t}-\sum_{q=1}^{Q} P_{E_{q}}^{t}-L^{t}\right) \\
& \left.-\nu^{t}\left(\tilde{P}_{R}^{t}-P_{R}^{t}-\sum_{j=1}^{J} P_{B_{j}}^{t}\right)\right\} .
\end{aligned}
$$

Then, the dual function can be written as

$$
\begin{aligned}
\mathcal{D}(\mathbf{z})= & \min _{\mathbf{x}} \mathcal{L}(\mathbf{x}, \mathbf{z}) \\
& \text { s.t. } 8 \mathrm{~b}-8 \mathrm{dd}, 88 \mathrm{fb}-8 \mathrm{~m}
\end{aligned}
$$

and the dual problem is given by

$$
\begin{array}{cl}
\max & \mathcal{D}\left(\left\{\mu^{t}\right\},\left\{\lambda^{t}\right\},\left\{\nu^{t}\right\}\right) \\
\text { s.t. } & \mu^{t} \geq 0, \lambda^{t}, \nu^{t} \in \mathbb{R}, t \in \mathcal{T} .
\end{array}
$$


The subgradient method will be employed to obtain the optimal multipliers and power schedules. The iterative process is described next, followed by its distributed implementation.

1) Subgradient Iterations: The subgradient method amounts to running the recursions [25, Sec. 6.3]

$$
\begin{aligned}
& \mu^{t}(k+1)=\left[\mu^{t}(k)+a g_{\mu^{t}}(k)\right]^{+} \\
& \lambda^{t}(k+1)=\lambda^{t}(k)+a g_{\lambda^{t}}(k) \\
& \nu^{t}(k+1)=\nu^{t}(k)+a g_{\nu^{t}}(k)
\end{aligned}
$$

where $k$ is the iteration index; $a>0$ is a constant stepsize; while $g_{\mu^{t}}(k), g_{\lambda^{t}}(k)$, and $g_{\nu^{t}}(k)$ denote the subgradients of the dual function with respect to $\mu^{t}(k), \lambda^{t}(k)$, and $\nu^{t}(k)$, respectively. These subgradients can be expressed in the following simple forms

$$
\begin{aligned}
g_{\mu^{t}}(k)= & \mathrm{SR}^{t}-\sum_{m=1}^{M}\left(P_{G_{m}}^{\max }-P_{G_{m}}^{t}(k)\right) \\
g_{\lambda^{t}}(k)= & L^{t}+\sum_{n=1}^{N} P_{D_{n}}^{t}(k)+\sum_{q=1}^{Q} P_{E_{q}}^{t}(k) \\
& -\sum_{m=1}^{M} P_{G_{m}}^{t}(k)-P_{R}^{t}(k) \\
g_{\nu^{t}}(k)= & P_{R}^{t}(k)+\sum_{j=1}^{J} P_{B_{j}}^{t}(k)-\tilde{P}_{R}^{t}(k)
\end{aligned}
$$

where $P_{G_{m}}^{t}(k), P_{D_{n}}^{t}(k), P_{E_{q}}^{t}(k), P_{B_{j}}^{t}(k), P_{R}^{t}(k)$, and $\tilde{P}_{R}^{t}(k)$ are given by (16) 20 .

Iterations are initialized with arbitrary $\lambda^{t}(0), \nu^{t}(0) \in \mathbb{R}$, and $\mu^{t}(0) \geq 0$. The iterates are guaranteed to converge to a neighborhood of the optimal multipliers [25, Sec. 6.3]. The size of the neighborhood is proportional to the stepsize, and can therefore be controlled by the stepsize.

When the primal objective is not strictly convex, a primal averaging procedure is necessary to obtain the optimal power schedules, which are then given by

$$
\overline{\mathbf{x}}(k)=\frac{1}{k} \sum_{j=0}^{k-1} \mathbf{x}(j)=\frac{1}{k} \mathbf{x}(k-1)+\frac{k-1}{k} \overline{\mathbf{x}}(k-1) .
$$

The running averages can be recursively computed as in 21, and are also guaranteed to converge to a neighborhood of the optimal solution [26]. Note that other convergence-guaranteed stepsize rules and primal averaging methods can also be utilized; see [27] for detailed discussions.

2) Distributed Implementation: The form of the subgradient iterations easily lends itself to a distributed implementation utilizing the control and communication capabilities of a typical microgrid.

Specifically, the MGEM maintains and updates the Lagrange multipliers via (14). The LCs of conventional generation, dispatchable loads, storage units, and RES solve subproblems (16-20), respectively. These subproblems can be solved if the MGEM sends the current multiplier iterates $\mu^{t}(k), \lambda^{t}(k)$, and $\nu^{t}(k)$ to the LCs. The LCs send back to the MGEM the quantities $\sum_{m=1}^{M} P_{G_{m}}^{t}(k), \sum_{n=1}^{N} P_{D_{n}}^{t}(k)$, $\sum_{q=1}^{Q} P_{E_{q}}^{t}(k), \sum_{j=1}^{J} P_{B_{j}}^{t}(k), P_{R}^{t}(k)$, and $\tilde{P}_{R}^{t}(k)$ which are in turn used to form the subgradients according to [15]. The distributed algorithm using dual decomposition is tabulated as Algorithm 1 and the interactive process of message passing is illustrated in Fig. 2

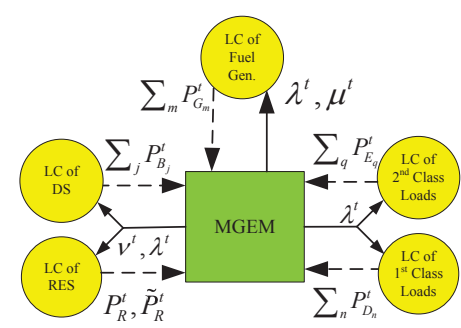

Fig. 2. Decomposition and message exchange.

$$
\begin{aligned}
& \left\{P_{G_{m}}^{t}(k)\right\}_{t=1}^{T} \in \underset{\left\{P_{G m}^{t}\right\}}{\arg \min }\left\{\sum_{t=1}^{T}\left(C_{m}^{t}\left(P_{G_{m}}^{t}\right)+\left(\mu^{t}(k)-\lambda^{t}(k)\right) P_{G_{m}}^{t}\right)\right\} \\
& \left\{P_{D_{n}}^{t}(k)\right\}_{t=1}^{T} \in \underset{\left\{P_{D_{n}}^{t}\right\}}{\arg \min }\left\{\sum_{t=1}^{T}\left(\lambda^{t}(k) P_{D_{n}}^{t}-U_{D_{n}}^{t}\left(P_{D_{n}}^{t}\right)\right)\right\} \\
& \left\{P_{E_{q}}^{t}(k)\right\}_{t=1}^{T} \in \underset{\left\{P_{E_{q}}^{t}\right\}}{\arg \min }\left\{\sum_{t=1}^{T}\left(\lambda^{t}(k) P_{E_{q}}^{t}-U_{E_{q}}^{t}\left(P_{E_{q}}^{t}\right)\right)\right\} \\
& \left\{P_{B_{j}}^{t}(k)\right\}_{t=1}^{T} \in \underset{\left\{P_{B_{j}}^{t}, B_{j}^{t}\right\}}{\arg \min }\left\{\sum_{t=1}^{T}\left(\nu^{t}(k) P_{B_{j}}^{t}+H_{j}^{t}\left(B_{j}^{t}\right)\right)\right\} \\
& \text { s.t. } 81-81 \\
& \left\{P_{R}^{t}(k), \tilde{P}_{R}^{t}(k)\right\}_{t=1}^{T} \in \underset{\substack{\left\{P_{R}^{t}, \tilde{P}_{R}^{t}\right\} \\
\text { s.t. } 8 \mathrm{~mm}}}{\arg \min }\left\{\sum_{t=1}^{T}\left(\left(\nu^{t}(k)-\lambda^{t}(k)\right) P_{R}^{t}\right)+G\left(\left\{\tilde{P}_{R}^{t}\right\}\right)-\sum_{t=1}^{T} \nu^{t}(k) \tilde{P}_{R}^{t}\right\}
\end{aligned}
$$



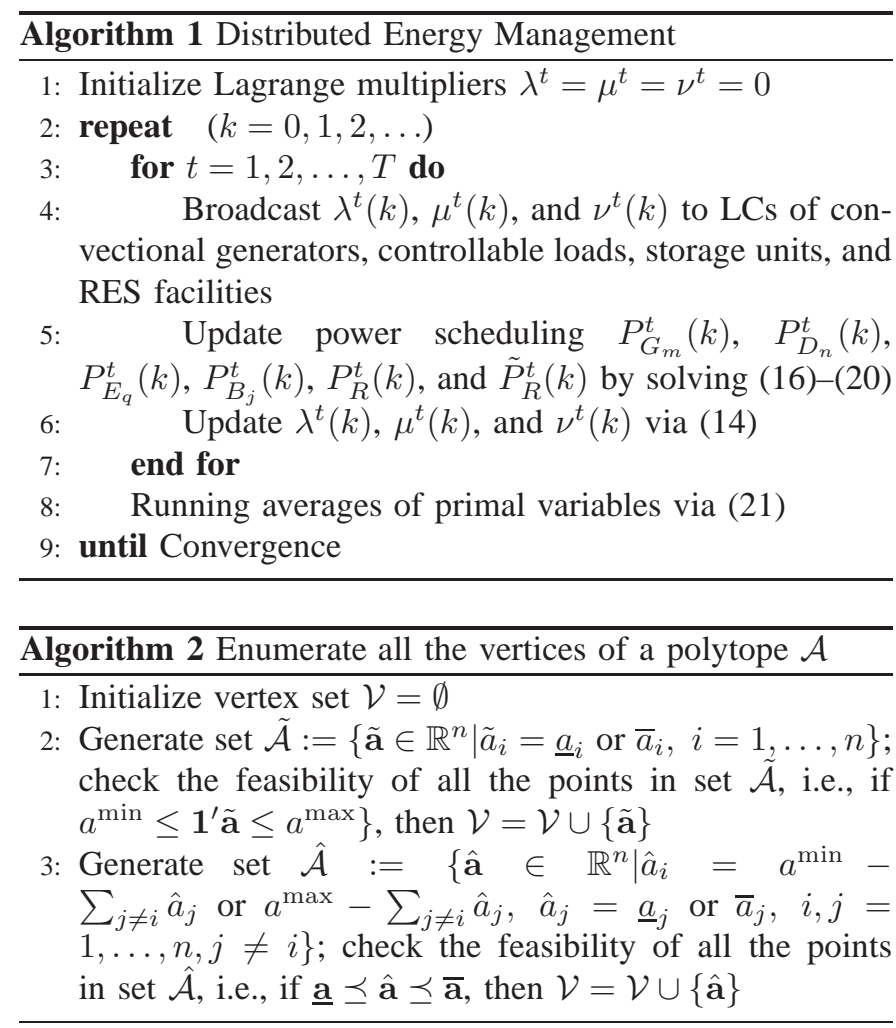

\section{B. Solving the LC Subproblems}

This subsection shows how to solve each subproblem (16)200. Specifically, $C_{m}^{t}(\cdot),-U_{D_{n}}^{t}(\cdot),-U_{E_{q}}^{t}(\cdot)$, and $H_{j}^{t}(\cdot)$ are chosen either convex piece-wise linear or smooth convex quadratic. Correspondingly, the first four subproblems 16(19) are essentially linear programs (LPs) or quadratic programs (QPs), which can be solved efficiently. Therefore, the main focus is on solving (20).

The optimal solution of $P_{R}^{t}(k)$ in 20 is easy to obtain as

$$
P_{R}^{t}(k)= \begin{cases}P_{R}^{\min }, & \text { if } \nu^{t}(k) \geq \lambda^{t}(k) \\ P_{R}^{\max }, & \text { if } \nu^{t}(k)<\lambda^{t}(k) .\end{cases}
$$

However, due to the absolute value operator and the maximization over $\mathbf{w}$ in the definition of $G\left(\left\{\tilde{P}_{R}^{t}\right\}\right)$, subproblem (20) is a convex nondifferentiable problem in $\left\{\tilde{P}_{R}^{t}\right\}$, which can be challenging to solve. As a state-of-the-art technique for convex nondifferentiable optimization problems [25, Ch. 6], the bundle method is employed to obtain $\left\{\tilde{P}_{R}^{t}(k)\right\}$.

Upon defining

$$
\tilde{G}\left(\left\{\tilde{P}_{R}^{t}\right\}\right):=G\left(\left\{\tilde{P}_{R}^{t}\right\}\right)-\sum_{t=1}^{T} \nu^{t}(k) \tilde{P}_{R}^{t}
$$

the subgradient of $\tilde{G}\left(\left\{\tilde{P}_{R}^{t}\right\}\right)$ with respect to $\tilde{P}_{R}^{t}$ needed for the bundle method can be obtained by the generalization of Danskin's Theorem [25, Sec. 6.3] as

$$
\partial \tilde{G}\left(\left\{\tilde{P}_{R}^{t}\right\}\right)= \begin{cases}\alpha^{t}-\nu^{t}(k), & \text { if } \tilde{P}_{R}^{t} \geq \sum_{i=1}^{I}\left(W_{i}^{t}\right)^{*} \\ \beta^{t}-\nu^{t}(k), & \text { if } \tilde{P}_{R}^{t}<\sum_{i=1}^{I}\left(W_{i}^{t}\right)^{*}\end{cases}
$$

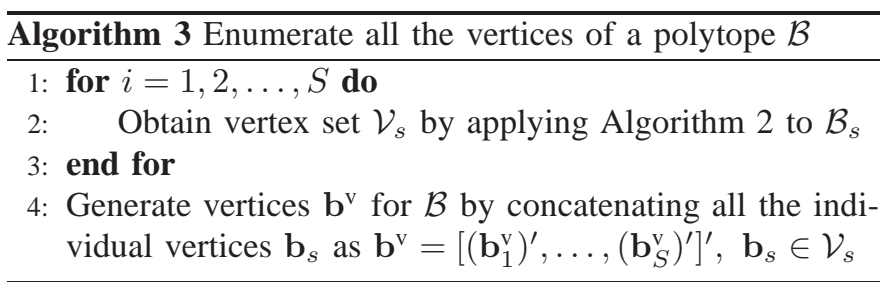

where for given $\left\{\tilde{P}_{R}^{t}\right\}$ it holds that

$\mathbf{w}^{*} \in \underset{\mathbf{w} \in \mathcal{W}}{\arg \max }\left\{\sum_{t=1}^{T}\left(\delta^{t}\left|\tilde{P}_{R}^{t}-\sum_{i=1}^{I} W_{i}^{t}\right|+\gamma^{t}\left(\tilde{P}_{R}^{t}-\sum_{i=1}^{I} W_{i}^{t}\right)\right)\right\}$.

With $\mathbf{p}:=\left[\tilde{P}_{R}^{1}, \ldots, \tilde{P}_{R}^{T}\right]$, the bundle method generates a sequence $\left\{\mathbf{p}_{\ell}\right\}$ with guaranteed convergence to the optimal $\left\{\tilde{P}_{R}^{t}(k)\right\}$; see e.g., [28], [25, Ch. 6]. The iterate $\mathbf{p}_{\ell+1}$ is obtained by minimizing a polyhedral approximation of $\tilde{G}(\mathbf{p})$ with a quadratic proximal regularization as follows

$$
\mathbf{p}_{\ell+1}:=\underset{\mathbf{p} \in \mathbb{R}^{T}}{\arg \min }\left\{\hat{G}_{\ell}(\mathbf{p})+\frac{\rho_{\ell}}{2}\left\|\mathbf{p}-\mathbf{y}_{\ell}\right\|^{2}\right\}
$$

where $\hat{G}_{\ell}(\mathbf{p}):=\max \left\{\tilde{G}\left(\mathbf{p}_{0}\right)+\mathbf{g}_{0}^{\prime}\left(\mathbf{p}-\mathbf{p}_{0}\right), \ldots, \tilde{G}\left(\mathbf{p}_{\ell}\right)+\right.$ $\left.\mathbf{g}_{\ell}^{\prime}\left(\mathbf{p}-\mathbf{p}_{\ell}\right)\right\} ; \mathbf{g}_{\ell}$ is the subgradient of $\tilde{G}(\mathbf{p})$ evaluated at the point $\mathbf{p}=\mathbf{p}_{\ell}$, which is calculated according to (24); proximity weight $\rho_{\ell}$ is to control stability of the iterates; and the proximal center $\mathbf{y}_{\ell}$ is updated according to a query for descent

$$
\mathbf{y}_{\ell+1}=\left\{\begin{array}{cc}
\mathbf{p}_{\ell+1}, & \text { if } \tilde{G}\left(\mathbf{y}_{\ell}\right)-\tilde{G}\left(\mathbf{p}_{\ell+1}\right) \geq \theta \eta_{\ell} \\
\mathbf{y}_{\ell}, & \text { otherwise }
\end{array}\right.
$$

where $\eta_{\ell}=\tilde{G}\left(\mathbf{y}_{\ell}\right)-\left(\hat{G}_{\ell}\left(\mathbf{p}_{\ell+1}\right)+\frac{\rho \ell}{2}\left\|\mathbf{p}_{\ell+1}-\mathbf{y}_{\ell}\right\|^{2}\right), \theta \in$ $(0,1)$.

It is worth mentioning that is essentially a QP over a simplex in the dual space, which is efficiently solvable by practical optimization algorithms. The corresponding transformation is shown in Appendix \for the interested readers.

Algorithms for solving (25) depend on the form of the uncertainty set $\mathcal{W}$, and are elaborated next.

\section{Vertex Enumerating Algorithms}

In order to obtain $\mathrm{w}^{*}$, the convex nondifferentiable function in (25) should be maximized over $\mathcal{W}$. This is generally an NPhard convex maximization problem. However, for the specific problem here, the special structure of the problem can be utilized to obtain a computationally efficient approach.

Specifically, the global solution is attained at the extreme points of the polytope [25, Sec. 2.4]. Therefore, the objective in (25) can be evaluated at all vertices of $\mathcal{W}$ to obtain the global solution. Since there are only finitely many vertices, (25) can be solved in a finite number of steps.

For the polytopes $\mathcal{W}$ with special structure [cf. (4), (6)], characterizations of vertices are established in Propositions 3 and 4 Capitalizing on these propositions, vertex enumerating procedures are designed consequently, and are tabulated as Algorithms 2 and 3 .

Proposition 3. For a polytope $\mathcal{A}:=\left\{\mathbf{a} \in \mathbb{R}^{n} \mid \underline{\mathbf{a}} \preceq \mathbf{a} \preceq\right.$ $\left.\overline{\mathbf{a}}, a^{\min } \leq \mathbf{1}^{\prime} \mathbf{a} \leq a^{\max }\right\}, \mathbf{a}^{v} \in \mathcal{A}$ is a vertex (extreme point) 
TABLE I

GENERATING CAPACITIES, RAMPING LIMITS, AND COST COEFFICIENTS, THE UNITS OF $a_{m}$ AND $b_{m}$ ARE $\$ /(\mathrm{KWH})^{2}$ AND $\$ / \mathrm{KWH}$, RESPECTIVELY.

\begin{tabular}{c||c|c|c|c|c}
\hline Unit & $P_{G_{m}}^{\min }$ & $P_{G_{m}}^{\max }$ & $R_{m, \text { up (down) }}$ & $a_{m}$ & $b_{m}$ \\
\hline 1 & 10 & 50 & 30 & 0.006 & 0.5 \\
2 & 8 & 45 & 25 & 0.003 & 0.25 \\
3 & 15 & 70 & 40 & 0.004 & 0.3 \\
\hline
\end{tabular}

TABLE II

Class- 1 DisPatchable LOADS PARAMETERS. THE UNITS OF $c_{n}$ AND $d_{n}$ ARE $\$ /(\mathrm{KWH})^{2}$ AND $\$ / \mathrm{KWH}$, RESPECTIVELY.

\begin{tabular}{c||c|c|c|c|c|c}
\hline & Load 1 & Load 2 & Load 3 & Load 4 & Load 5 & Load 6 \\
\hline \hline$P_{D_{n}}^{\min }$ & 0.5 & 4 & 2 & 5.5 & 1 & 7 \\
$P_{D_{n}}^{\max }$ & 10 & 16 & 15 & 20 & 27 & 32 \\
\hline$c_{n}$ & -0.002 & -0.0017 & -0.003 & -0.0024 & -0.0015 & -0.0037 \\
$d_{n}$ & 0.2 & 0.17 & 0.3 & 0.24 & 0.15 & 0.37 \\
\hline
\end{tabular}

of $\mathcal{A}$ if and only if it has one of the following forms: i) $a_{i}^{v}=$ $\underline{a}_{i}$ or $\bar{a}_{i}$ for $i=1, \ldots, n$; or ii) $a_{i}^{v}=a^{\min }-\sum_{j \neq i} a_{j}^{v}$ or $a^{\max }-$ $\sum_{j \neq i} a_{j}^{v}, a_{j}^{v}=\underline{a}_{j}$ or $\bar{a}_{j}$, for $i, j=1, \ldots, n, j \neq i$.

Proof: See Appendix 【I-A.

Essentially, Proposition 3 verifies the geometric characterization of vertices. Since $\mathcal{W}$ is the part of a hyperrectangle (orthotope) between two parallel hyperplanes, its vertices can only either be the hyperrectangle's vertices which are not cut away, or, the vertices of the intersections of the hyperrectangle and the hyperplanes, which must appear in some edges of the hyperrectangle.

Next, the vertex characterization of a polytope in a Cartesian product formed by many lower-dimensional polytopes like $\mathcal{A}$ is established, which is needed for the uncertainty set (4).

Proposition 4. Assume $\mathbf{b} \in \mathbb{R}^{n}$ is divided into $S$ consecutive and non-overlapping blocks as $\mathbf{b}=\left[\mathbf{b}_{1}^{\prime}, \ldots, \mathbf{b}_{S}^{\prime}\right]^{\prime}$, where $\mathbf{b}_{s} \in$ $\mathbb{R}^{n_{s}}$ and $\sum_{s=1}^{S} n_{s}=n$. Consider a polytope $\mathcal{B}:=\{\mathbf{b} \in$ $\left.\mathbb{R}^{n} \mid \underline{\mathbf{b}} \preceq \mathbf{b} \preceq \overline{\mathbf{b}}, b_{s}^{\min } \leq \mathbf{1}_{n_{s}}^{\prime} \mathbf{b}_{s} \leq b_{s}^{\max }, s=1, \ldots, S\right\}$. Then $\mathbf{b}^{v}=\left[\left(\mathbf{b}_{1}^{v}\right)^{\prime}, \ldots,\left(\mathbf{b}_{S}^{v}\right)^{\prime}\right]^{\prime}$ is a vertex of $\mathcal{B}$ if and only if for $s=1, \ldots, S, \mathbf{b}_{s}^{v}$ is the vertex of a lower-dimensional polytope $\mathcal{B}_{s}:=\left\{\mathbf{b}_{s} \in \mathbb{R}^{n_{s}} \mid \underline{\mathbf{b}}_{s} \preceq \mathbf{b}_{s} \preceq \overline{\mathbf{b}}_{s}, b_{s}^{\min } \leq \mathbf{1}_{n_{s}}^{\prime} \mathbf{b}_{s} \leq b_{s}^{\max }\right\}$.

Proof: See Appendix

Algorithms 2 and 3 can be used to to generate the vertices of uncertainty sets (4) and (6) as described next.

i) For uncertainty set (4), first use Algorithm 2 to obtain the vertices corresponding to each sub-horizon $\mathcal{T}_{i, s}$ for all the RES facilities. Then, concatenate the obtained vertices to get the ones for each RES facility by Step 4 in Algorithm 3. Finally, run this step again to form the vertices of (44) by concatenating the vertices of each $\mathcal{W}_{i}$.

ii) For uncertainty sets (6), use Algorithm 2 to obtain the vertices for each sub-horizon $\mathcal{T}_{s}$. Note that concatenating step in Algorithm 3 is not needed in this case because problem 25] is decomposable across sub-horizons $\mathcal{T}_{s}$, $s=1, \ldots, S$, and can be independently solved accordingly.

After the detailed description of vertex enumerating procedures for RES uncertainty sets, a discussion on the complexity of solving 25] follows.
TABLE III

CLASS-2 DISPATCHABLE LOADS PARAMETERS

\begin{tabular}{c||c|c|c|c}
\hline & Load 1 & Load 2 & Load 3 & Load 4 \\
\hline \hline$P_{E_{q}}^{\min }$ & 0 & 0 & 0 & 0 \\
$P_{E_{q}}^{\max }$ & 1.2 & 1.55 & 1.3 & 1.7 \\
$E_{q}^{\max }$ & 5 & 5.5 & 4 & 8 \\
$S_{q}$ & $6 \mathrm{PM}$ & $7 \mathrm{PM}$ & $6 \mathrm{PM}$ & $6 \mathrm{PM}$ \\
$T_{q}$ & $12 \mathrm{AM}$ & $11 \mathrm{PM}$ & $12 \mathrm{AM}$ & $12 \mathrm{AM}$ \\
\hline
\end{tabular}

TABLE IV

LIMITS OF FORECASTED WIND POWER

\begin{tabular}{c||c|c|c|c|c|c|c|c}
\hline Slot & 1 & 2 & 3 & 4 & 5 & 6 & 7 & 8 \\
\hline \hline$W_{1}^{t}$ & 2.47 & 2.27 & 2.18 & 1.97 & 2.28 & 2.66 & 3.1 & 3.38 \\
$\bar{W}_{1}^{t}$ & 24.7 & 22.7 & 21.8 & 19.7 & 22.8 & 26.6 & 31 & 33.8 \\
$W_{2}^{t}$ & 2.57 & 1.88 & 2.16 & 1.56 & 1.95 & 3.07 & 3.44 & 3.11 \\
$\bar{W}_{2}^{t}$ & 25.7 & 18.8 & 21.6 & 15.6 & 19.5 & 30.7 & 34.4 & 31.1 \\
\hline
\end{tabular}

Remark 2. (Complexity of solving (25)). Vertex enumeration incurs exponential complexity because the number of vertices can increase exponentially with the number of variables and constraints [29, Ch. 2]. However, if the cardinality of each sub-horizon $\mathcal{T}_{s}$ is not very large (e.g., when 24 hours are partitioned into 4 sub-horizons each comprising 6 time slots), then the complexity is affordable. Most importantly, the vertices of $\mathcal{W}$ need only be listed once, before optimization.

\section{Numerical Tests}

In this section, numerical results are presented to verify the performance of the robust and distributed energy scheduler. The Matlab-based modeling package CVX [30] along with the solver MOSEK [31] are used to specify and solve the proposed robust energy management problem. The considered microgrid consists of $M=3$ conventional generators, $N=6$ class-1 dispatchable loads, $Q=4$ class- 2 dispatchable loads, $J=3$ storage units, and $I=2$ renewable energy facilities (wind farms). The time horizon spans $T=8$ hours, corresponding to the interval $4 \mathrm{PM}-12 \mathrm{AM}$. The generation costs $C_{m}\left(P_{G_{m}}\right)=a_{m} P_{G_{m}}^{2}+b_{m} P_{G_{m}}$ and the utilities of class1 elastic loads $U_{n}\left(P_{D_{n}}\right)=c_{n} P_{D_{n}}^{2}+d_{n} P_{D_{n}}$ are set to be quadratic and time-invariant. Generator parameters are given in Table [] while $\mathrm{SR}^{t}=10 \mathrm{kWh}$. The relevant parameters of two classes of dispatchable loads are listed in Tables $\Pi$ and (see also [27]). The utility of class-2 loads is $U_{E_{q}}^{t}\left(P_{E_{q}}^{t}\right):=\pi_{q}^{t} P_{E_{q}}^{t}$ with weights $\pi_{q}^{t}=4,3.5, \ldots, 1,0.5$ for $t=4 \mathrm{PM}, \ldots, 11 \mathrm{PM}$ and $q \in \mathcal{Q}$.

Three batteries have capacity $B_{j}^{\max }=30 \mathrm{kWh}$ (similar to [5]). The remaining parameters are $P_{B_{j}}^{\min }=-10 \mathrm{kWh}$, $P_{B_{j}}^{\max }=10 \mathrm{kWh}, B_{j}^{0}=B_{j}^{\min }=5 \mathrm{kWh}$, and $\eta_{j}=0.95$, for all $j \in \mathcal{J}$. The battery costs $H_{j}^{t}\left(B_{j}^{t}\right)$ are set to zero. The joint uncertainty model with $S=1$ is considered for $\mathcal{W}$ [cf. [6] ], where $W_{1}^{\min }=40 \mathrm{kWh}$, and $W_{1}^{\max }=360 \mathrm{kWh}$. In order to obtain $\underline{W}_{i}^{t}$ and $\bar{W}_{i}^{t}$ listed in Table IV MISO day-ahead wind forecast data [32] are rescaled to the order of $1 \mathrm{kWh}$ to $40 \mathrm{kWh}$, which is a typical wind power generation for a microgrid [33].

Similarly, the fixed load $L^{t}$ in Table $\nabla$ is a rescaled version of the cleared load provided by MISO's daily report [34]. For 
TABLE V

FIXED LOADS DEMAND AND TRANSACTION PRICES. THE UNITS OF $\alpha^{t}$ AND $\beta^{t}$ ARE $\phi / K W H$.

\begin{tabular}{l||l|l|l|l|l|l|l|l}
\hline Slot & 1 & 2 & 3 & 4 & 5 & 6 & 7 & 8 \\
\hline \hline$L^{t}$ & 57.8 & 58.4 & 64 & 65.1 & 61.5 & 58.8 & 55.5 & 51 \\
\hline (Case A) & & & & & & & & \\
$\alpha^{t}$ & 2.01 & 2.2 & 3.62 & 6.6 & 5.83 & 3.99 & 2.53 & 2.34 \\
$\beta^{t}$ & 1.81 & 1.98 & 3.26 & 5.94 & 5.25 & 3.59 & 2.28 & 2.11 \\
\hline (Case B) & & & & & & & & \\
$\alpha^{t}$ & 40.2 & 44 & 72.4 & 132 & 116.6 & 79.8 & 50.6 & 46.8 \\
$\beta^{t}$ & 36.18 & 39.6 & 65.16 & 118.8 & 104.94 & 71.82 & 45.54 & 42.12 \\
\hline
\end{tabular}

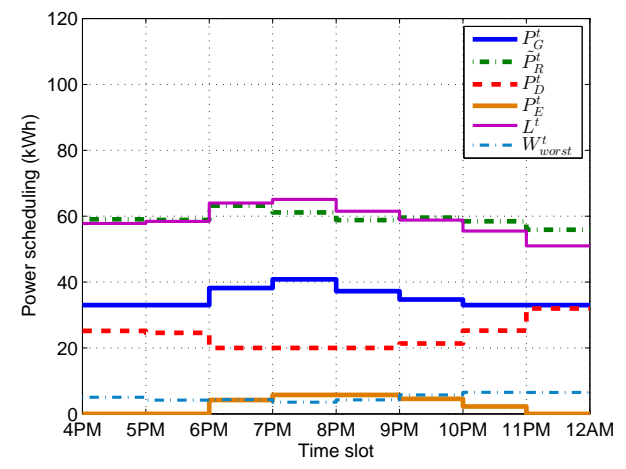

Fig. 3. Optimal power schedules: Case A.

the transaction prices, two different cases are studied as given in Table $\mathrm{V}$, where $\left\{\alpha^{t}\right\}$ in Case A are real-time prices of the Minnesota hub in MISO's daily report. To evaluate the effect of high transaction prices, $\left\{\alpha^{t}\right\}$ in Case B is set as 20 times of that in Case A. For both cases, $\beta^{t}=0.9 \alpha^{t}$, which satisfies the convexity condition for (P1) given in Proposition 1

The optimal microgrid power schedules of two cases are shown in Figs. 3 and 4 The stairstep curves include $P_{G}^{t}:=$ $\sum_{m} P_{G_{m}}^{t}, P_{D}^{t}:=\sum_{n} P_{D_{n}}^{t}$, and $P_{E}^{t}:=\sum_{q} P_{E_{q}}^{t}$ denoting the total conventional power generation, and total elastic demand for classes 1 and 2, respectively, which are the optimal solutions of (P2). Quantity $W_{\text {worst }}^{t}$ denotes the total worst-case wind energy at slot $t$, which is the optimal solution of 25) with optimal $\tilde{P}_{R}^{t}$.

A common observation from Figs. 3 and 4 is that the total conventional power generation $P_{G}^{t}$ varies with the same trend across $t$ as the fixed load demand $L^{t}$, while the class- 1 elastic load exhibits the opposite trend. Because the conventional generation and the power drawn from the main grid are limited, the optimal scheduling by solving (P2) dispatches less power for $P_{D}^{t}$ when $L^{t}$ is large (from $6 \mathrm{PM}$ to $10 \mathrm{PM}$ ), and vice versa. This behavior indeed reflects the load shifting ability of the proposed design for the microgrid energy management.

Furthermore, by comparing two cases in Figs. 3 and 4 it is interesting to illustrate the effect of the transaction prices. Remember that the difference between $\tilde{P}_{R}^{t}$ and $W_{\text {worst }}^{t}$ is the shortage power needed to purchase (if positive) or the surplus power to be sold (if negative), Figs. 3 shows that the microgrid always purchases energy from the main grid because $\tilde{P}_{R}^{t}$ is more than $W_{\text {worst }}^{t}$. This is because for Case $\mathrm{A}$, the purchase price $\alpha^{t}$ is much lower than the marginal cost of the conventional generation (cf. Tables \and $\mathbf{V}$. The

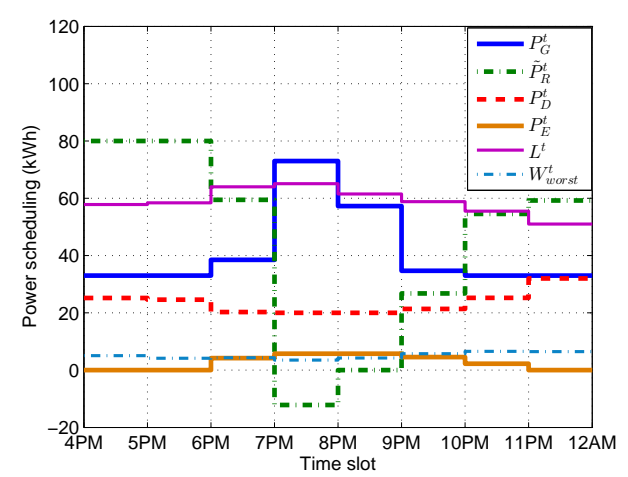

Fig. 4. Optimal power schedules: Case B.

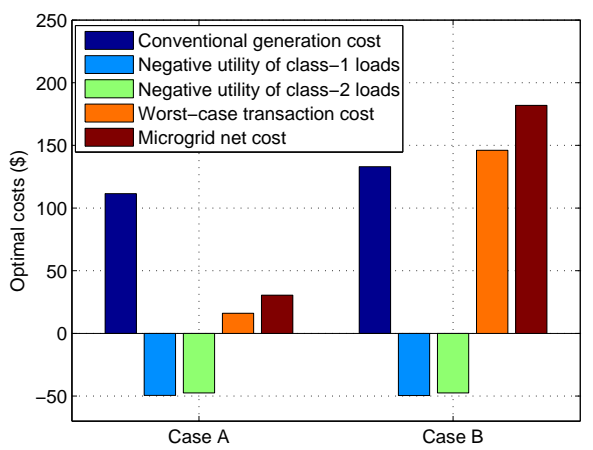

Fig. 5. Optimal costs: Case A and B.

economic scheduling decision is thus to reduce conventional generation while purchasing more power to keep the supplydemand balance. For Case B, since $\alpha^{t}$ is much higher than that in Case A, less power should be purchased which is reflected in the relatively small gap between $\tilde{P}_{R}^{t}$ and $W_{\text {worst }}^{t}$ across time slots. It can also be seen that $\tilde{P}_{R}^{t}$ is smaller than $W_{\text {worst }}^{t}$ from $7 \mathrm{PM}$ to 9PM, meaning that selling activity happens and is encouraged by the highest selling price $\beta^{t}$ in these slots across the entire time horizon. Moreover, selling activity results in the peak conventional generation from 7PM to 9PM. Fig. 5 compares the optimal costs for the two cases. It can be seen that the optimal costs of conventional generation and worstcase transaction of Case B are higher than those of Case A, which can be explained by the higher transaction prices and the resultant larger DG output for Case B.

The optimal power scheduling of class-2 elastic load is depicted in Fig. 6 for Case A. Due to the start time $S_{q}$ (cf. Table III), zero power is scheduled for the class-2 load 1,3 , and 4 from $4 \mathrm{PM}$ to $6 \mathrm{PM}$ while from $4 \mathrm{PM}$ to $7 \mathrm{PM}$ for the load 2. The decreasing trend for all such loads is due to the decreasing weights $\left\{\pi_{q}^{t}\right\}$ from $S_{q}$ to $T_{q}$, which is established from the fast charging motivation for the PHEVs, for example.

Figs. 7 depicts the optimal charging or discharging power of the DSs for Case B. Clearly, all DSs are discharging during the three slots of 7PM, 8PM, and 9PM. This results from the motivation of selling more or purchasing less power because both purchase and selling prices are very high during these slots (cf. Table $\mathrm{V}$ ). The charging (discharging) activity can also 


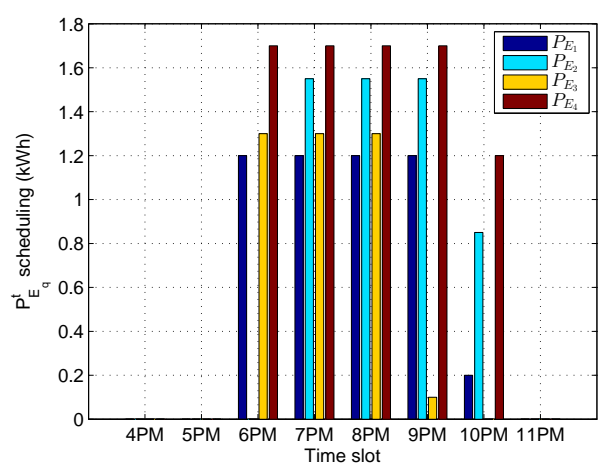

Fig. 6. Optimal power schedule for $P_{E_{q}}^{t}$ : Case A.

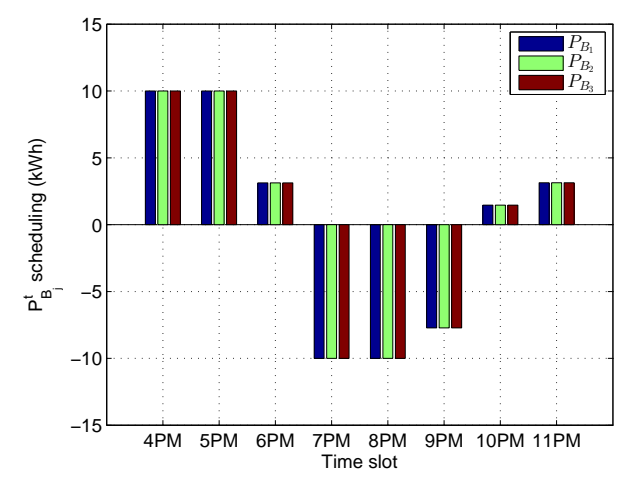

Fig. 7. Optimal power schedule for $P_{B_{j}}^{t}$ : Case B.

be reflected by the stored energy of the battery devices shown in Fig. 8 . Note that, starting from the initial energy $5 \mathrm{kWh}$ at 4PM, the optimal stored energy of all units are scheduled to have $5 \mathrm{kWh}$ at $12 \mathrm{AM}$, which satisfies the minimum stored energy requirement for the next round of scheduling time horizons.

Finally, Fig. 9 shows the effect of different selling prices $\left\{\beta^{t}\right\}$ on the optimal energy costs, where Case B is studied with fixed purchase prices $\left\{\alpha^{t}\right\}$. It can be clearly seen that the net cost decreases with the increase of the selling-to-purchaseprice ratio $\beta^{t} / \alpha^{t}$. When this ratio increases, the microgrid has a higher margin for revenue from the transaction mechanism, which yields the reduced worst-case transaction cost.

\section{CONClusions ANd Future Work}

A distributed energy management approach was developed tailored for microgrids with high penetration of renewable energy sources. By introducing the notion of committed renewable energy, a novel model was introduced to deal with the challenging constraint of the supply-demand balance raised by the intermittent nature of renewable energy sources. Not only the conventional generation costs, utilities of the adjustable loads, and distributed storage costs were accounted for, but also the worst-case transaction cost was included in the objective. To schedule power in a distributed fashion, the dual decomposition method was utilized to decompose the original problem into smaller subproblems solved by the LCs

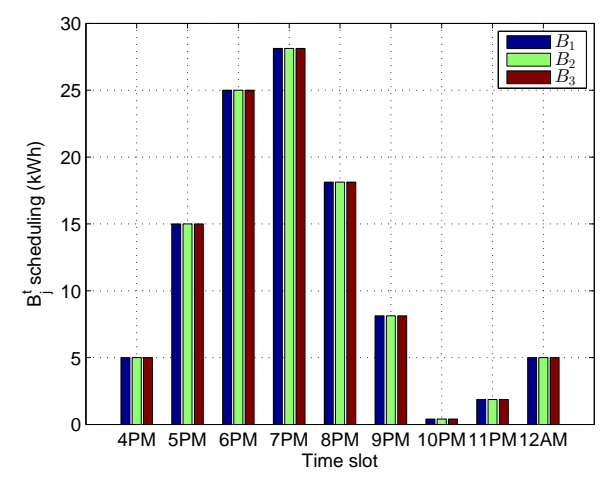

Fig. 8. Optimal power schedule for $B_{j}^{t}$ : Case B.

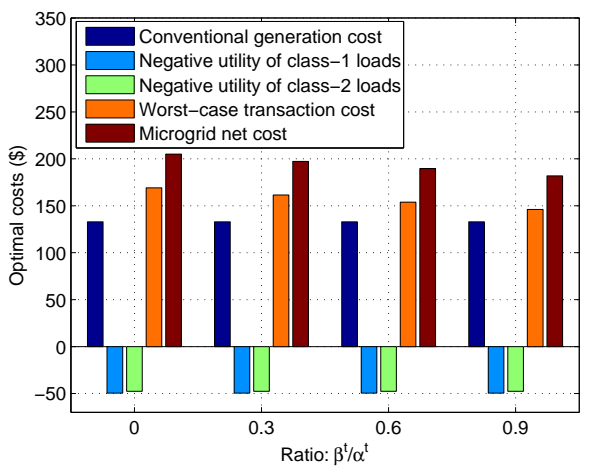

Fig. 9. Optimal costs: Case B.

of conventional generators, dispatchable loads, DS units and the RES.

A number of interesting research directions open up towards extending the model and approach proposed in this paper. Some classical but fundamental problems, such as the optimal power flow (OPF) and the unit commitment (UC) problems are worth re-investigating with the envisaged growth of RES usage in microgrids.

\section{APPENDIX I \\ ENHANCING THE BundLE Method}

Using an auxiliary variable $r$, 26) can be re-written as

$$
\begin{array}{cl}
\min _{\mathbf{p}, r} & r+\frac{\rho_{\ell}}{2}\left\|\mathbf{p}-\mathbf{y}_{\ell}\right\|^{2} \\
\text { s.t. } & \tilde{G}\left(\mathbf{p}_{i}\right)+\mathbf{g}_{i}^{\prime}\left(\mathbf{p}-\mathbf{p}_{i}\right) \leq r, i=0,1, \ldots, \ell .
\end{array}
$$

Introducing multipliers $\boldsymbol{\xi} \in \mathbb{R}_{+}^{\ell+1}$, the Lagrangian is given as

$$
\begin{aligned}
\mathcal{L}(r, \mathbf{p}, \boldsymbol{\xi})= & \left(1-\sum_{i=0}^{\ell+1} \xi_{i}\right) r+\frac{\rho_{\ell}}{2}\left\|\mathbf{p}-\mathbf{y}_{\ell}\right\|^{2} \\
& +\sum_{i=0}^{\ell+1} \xi_{i}\left(\tilde{G}\left(\mathbf{p}_{i}\right)+\mathbf{g}_{i}^{\prime}\left(\mathbf{p}-\mathbf{p}_{i}\right)\right)
\end{aligned}
$$

Optimality condition on $\mathbf{p}$, i.e., $\nabla_{\mathbf{p}} \mathcal{L}(r, \mathbf{p}, \boldsymbol{\xi})=\mathbf{0}$, yields

$$
\mathbf{p}^{*}=\mathbf{y}_{\ell}-\frac{1}{\rho_{\ell}} \sum_{i=0}^{\ell+1} \xi_{i} \mathbf{g}_{i} \text {. }
$$


Substituting (30) into (29), the dual of (28) is

$$
\begin{aligned}
& \max _{\boldsymbol{\xi}}-\frac{1}{2 \rho_{\ell}}\left\|\sum_{i=0}^{\ell+1} \xi_{i} \mathbf{g}_{i}\right\|^{2}+\sum_{i=0}^{\ell+1} \xi_{i}\left(\tilde{G}\left(\mathbf{p}_{i}\right)+\mathbf{g}_{i}^{\prime}\left(\mathbf{y}_{\ell}-\mathbf{p}_{i}\right)\right) \\
& \text { s.t. } \quad \boldsymbol{\xi} \succeq \mathbf{0}, \mathbf{1}^{\prime} \boldsymbol{\xi}=1
\end{aligned}
$$

where $\mathbf{1}$ is the all-ones vector.

Note that 31 is essentially a QP over the simplex in $\mathbb{R}^{\ell+1}$, which can be solved very efficiently.

\section{APPENDIX II \\ ProOFS OF PROPOSITIONS}

To prove Propositions 3 and 4 the following lemma is needed, which shows sufficient and necessary conditions for a point to be a vertex of a polytope represented as a linear system [35, Sec. 3.5].

Lemma 1. For a polytope $\mathcal{P}:=\left\{\mathbf{x} \in \mathbb{R}^{n} \mid \mathbf{A} \mathbf{x} \preceq \mathbf{c}\right\}$, a point $\mathbf{v} \in \mathcal{P}$ is a vertex if and only if there exists a subsystem $\tilde{\mathbf{A}} \mathbf{x} \preceq \tilde{\mathbf{c}}$ of $\mathbf{A} \mathbf{x} \preceq \mathbf{c}$ so that $\operatorname{rank}(\tilde{\mathbf{A}})=n$ and $\mathbf{v}$ is the unique (feasible) solution of $\tilde{\mathbf{A}} \mathbf{v}=\tilde{\mathbf{c}}$.

\section{A. Proof of Proposition 3}

The polytope $\mathcal{A}:=\left\{\mathbf{a} \in \mathbb{R}^{n} \mid \underline{\mathbf{a}} \preceq \mathbf{a} \preceq \overline{\mathbf{a}}, a^{\min } \leq \mathbf{1}^{\prime} \mathbf{a} \leq\right.$ $\left.a^{\max }\right\}$ can be re-written as $\mathcal{A}:=\left\{\mathbf{a} \in \mathbb{R}^{n} \mid \mathbf{A a} \preceq \mathbf{c}\right\}$, where $\mathbf{A}:=\left[\mathbf{I}_{n \times n},-\mathbf{I}_{n \times n}, \mathbf{1},-\mathbf{1}\right]^{\prime}$ and $\mathbf{c}:=\left[\overline{\mathbf{a}}^{\prime},-\underline{\mathbf{a}}^{\prime}, a^{\max },-a^{\mathrm{min}}\right]^{\prime}$. By Lemma 1 enumerating vertices of $\mathcal{A}$ is equivalent to finding all feasible solutions of the linear subsystems $\tilde{\mathbf{A}} \mathbf{a}=\tilde{\mathbf{c}}$, such that rank- $n$ matrix $\tilde{\mathbf{A}}$ is constructed by extracting rows of $\mathbf{A}$. It can be seen that such full column-rank matrix $\tilde{\mathbf{A}}$ can only have two forms (with row permutation if necessary): i) $\tilde{\mathbf{A}}_{1}=\operatorname{diag}(\mathbf{d})$ with $d_{i} \in\{-1,1\}, i=1, \ldots, n$; ii) $\tilde{\mathbf{A}}_{2}(i,:)=$ $\pm \mathbf{1}^{\prime}, i=1, \ldots, n$, and $\tilde{\mathbf{A}}_{2}(j,:)=\tilde{\mathbf{A}}_{1}(j,:), \forall j \neq i$. Basically, $\tilde{\mathbf{A}}_{1}$ is constructed by choosing $n$ vectors as a basis of $\mathbb{R}^{n}$ from the first $2 n$ rows of $\mathbf{A}$. Substituting any row of $\tilde{\mathbf{A}}_{1}$ with $\pm \mathbf{1}^{\prime}$, forms $\tilde{\mathbf{A}}_{2}$. Finally, by solving all the linear subsystems of the form $\tilde{\mathbf{A}}_{k} \mathbf{a}=\tilde{\mathbf{c}}_{k}$, for $k=1,2$, Proposition 3 follows readily.

\section{B. Proof of Proposition 4}

The polytope $\mathcal{B}:=\left\{\mathbf{b} \in \mathbb{R}^{n} \mid \underline{\mathbf{b}} \preceq \mathbf{b} \preceq \overline{\mathbf{b}}, b_{s}^{\min } \leq \mathbf{1}_{n_{s}}^{\prime} \mathbf{b}_{s} \leq\right.$ $\left.b_{s}^{\max }, s=1, \ldots, S\right\}$ can be re-written as $\mathcal{B}:=\left\{\mathbf{b} \in \mathbb{R}^{n} \mid \mathbf{B b} \preceq\right.$ $\mathbf{c}\}$, where $\mathbf{B}:=\operatorname{diag}\left(\mathbf{B}_{1}, \ldots, \mathbf{B}_{S}\right), \mathbf{c}:=\left[\mathbf{c}_{1}^{\prime}, \ldots, \mathbf{c}_{S}^{\prime}\right]^{\prime}, \mathbf{B}_{s}:=$ $\left[\mathbf{I}_{n_{s} \times n_{s}},-\mathbf{I}_{n_{s} \times n_{s}}, \mathbf{1},-\mathbf{1}\right]^{\prime}$, and $\mathbf{c}_{s}:=\left[\overline{\mathbf{a}}_{s}^{\prime},-\underline{\mathbf{a}}_{s}^{\prime}, b_{s}^{\max },-b_{s}^{\min }\right]^{\prime}$ for $s=1, \ldots, S$.

Similarly by Lemma 1, all the vertices of $\mathcal{B}$ can be enumerated by solving $\tilde{\mathbf{B}} \mathbf{b}=\tilde{\mathbf{c}}$, where the rank- $n$ matrix $\tilde{\mathbf{B}}$ is formed by extracting rows of $\mathbf{B}$. Due to the block diagonal structure of $\mathbf{B}$, it can be seen that the only way to find its $n$ linear independent rows is to find $n_{s}$ linear independent vectors from the rows corresponding to $\mathbf{B}_{s}$ for $s=1, \ldots, S$. In other words, the vertices $\mathbf{b}^{\mathbf{v}}$ can be obtained by concatenating all the individual vertices $\mathbf{b}_{s}$ as stated in Proposition 4

\section{REFERENCES}

[1] N. Hatziargyriou, H. Asano, R. Iravani, and C. Marnay, "Microgrids: An overview of ongoing research, development, and demonstration projects," IEEE Power \& Energy Mag., vol. 5, no. 4, pp. 78-94, JulyAug. 2007.

[2] P. Stluka, D. Godbole, and T. Samad, "Energy management for buildings and microgrids," in Proc. of the 50th IEEE Conf. on Decision and Control and European Control Conf., Orlando, FL, Dec. 12-15, 2011.

[3] J. Hetzer, C. Yu, and K. Bhattarai, "An economic dispatch model incorporating wind power," IEEE Trans. on Energy Conver., vol. 23 , no. 2, pp. 603-611, June 2008

[4] X. Liu and W. Xu, "Economic load dispatch constrained by wind power availability: A here-and-now approach," IEEE Trans. on Sustainable Energy, vol. 1, no. 1, pp. 2-9, Apr. 2010.

[5] X. Guan, Z. Xu, and Q.-S. Jia, "Energy-efficient buildings facilitated by microgrid," IEEE Trans. on Smart Grid, vol. 1, no. 3, pp. 243-252, Dec. 2010.

[6] D. Bertsimas, E. Litvinov, X. Sun, J. Zhao, and T. Zheng, "Adaptive robust optimization for the security constrained unit commitment problem," Mar. 2011, [Online]. Available: http://web.mit.edu/sunx/www/ Adaptive_Robust_UC_Revision.pdf.

[7] L. Jiang and S. H. Low, "Real-time demand response with uncertain renewable energy in smart grid," in Proc. of the 49th Allerton Conf. on Comm., Control, and Computing, Monticello, IL, Sept. 2011, pp. $1334-1341$.

[8] L. Zhao and B. Zeng, "Robust unit commitment problem with demand response and wind energy," Univ. of S. Florida, Tech. Rep., Oct. 2010, [Online]. Available: http://www.optimization-online.org/DB_ FILE/2010/11/2784.pdf

[9] C. Jin and P. K. Ghosh, "Coordinated usage of distributed sources for energy cost saving in micro-grid," in Proc. of the 43rd North American Power Symposium (NAPS), Boston, MA, Aug. 4-6, 2011.

[10] A. D. Domínguez-García and C. N. Hadjicostis, "Distributed algorithms for control of demand response and distributed energy resources," in Proc. of the 50th IEEE Control and Decision Conf., Orlando, FL, Dec. 2011.

[11] Y. Zhang, N. Gatsis, and G. B. Giannakis, "Robust distributed energy management for microgrids with renewables," in Proc. of 3rd Intl. Conf. on Smart Grid Commun., Tainan, Taiwan, Nov. 5-8, 2012.

[12] L. Chen, N. Li, S. H. Low, and J. C. Doyle, "Two market models for demand response in power networks," in Proc. 1st IEEE Intl. Conf. Smart Grid Communications, Gaithersburg, MD, Oct. 2010, pp. 397-402.

[13] A.-H. Mohsenian-Rad, V. S. W. Wong, J. Jatskevich, R. Schober, and A. Leon-Garcia, "Autonomous demand side management based on game-theoretic energy consumption scheduling for the future smart grid," IEEE Trans. on Smart Grid, vol. 1, no. 3, pp. 320-331, Dec. 2010.

[14] P. Vytelingum, T. D. Voice, S. D. Ramchurn, A. Rogers, and N. R. Jennings, "Agent-based micro-storage management for the smart grid," in Proc. 9th Intl. Conf. Autonomous and Multiagent Systems, Toronto, Canada, May 2010, pp. 39-46.

[15] V. Alimisis and N. Hatziargyriou, "Evaluation of a hybrid power plant comprising used EV-batteries to complement wind power," IEEE Trans. on Sustainable Energy, vol. 4, no. 2, pp. 286-293, Apr. 2013.

[16] P. Pinson and G. Kariniotakis, "Conditional prediction intervals of wind power generation," IEEE Trans. on Power Syst., vol. 25, no. 4, pp. 1845 1856, Nov. 2010.

[17] Y. Zhang, N. Gatsis, and G. B. Giannakis, "Risk-constrained energy management with multiple wind farms," in Proc. of 4th IEEE Conf. Innovative Smart Grid Tech., Washington, D.C., Feb. 24-27, 2013.

[18] J. M. Morales, L. Baringo, A. J. Conejo, and R. Mínguez, "Probabilistic power flow with correlated wind sources," IET Generation, Transmission \& Distribution, vol. 4, no. 5, pp. 641-651, May 2010.

[19] J. A. Carta, P. Ramírez, and S. Velázquez, "A review of wind speed probability distributions used in wind energy analysis: Case studies in the canary islands," Renew. Sust. Energ. Rev., vol. 13, pp. 933-955, 2009.

[20] S. Boyd and L. Vandenberghe, Convex Optimization. U.K.: Cambridge University Press, 2004

[21] D. P. Bertsekas, Nonlinear Programming, 2nd ed. Belmont, MA: Athena Scientific, 1999.

[22] A. J. Conejo, E. Castillo, R. Mínguez, and R. García-Bertrand, Decomposition Techniques in Mathematical Programming: Engineering and Science Applications. Springer, 2006.

[23] D. Palomar and M. Chiang, "A tutorial on decomposition methods for network utility maximization," IEEE J. Sel. Areas Commun., vol. 46, no. 8, pp. 1439-1451, Aug. 2006. 
[24] M. Chiang, S. H. Low, A. R. Calderbank, and J. C. Doyle, "Layering as optimization decomposition: A mathematical theory of network architectures," Proc. of the IEEE, vol. 95, no. 1, pp. 255-312, Jan. 2007.

[25] D. P. Bertsekas, Convex Optimization Theory. Belmont, MA: Athena Scientific, 2009.

[26] A. Nedić and A. Ozdaglar, "Approximate primal solutions and rate analysis for dual subgradient methods," SIAM J. Optim., vol. 19, no. 4, pp. 1757-1780, 2009.

[27] N. Gatsis and G. B. Giannakis, "Residential load control: Distributed scheduling and convergence with lost AMI messages," IEEE Trans. on Smart Grid, vol. 3, no. 2, pp. 770-786, June 2012.

[28] S. Feltenmark and K. C. Kiwiel, "Dual application of proximal bundle methods, including Lagrange relaxation of nonconvex problems," SIAM J. Optim., vol. 10, no. 3, pp. 697-721, Feb./Mar. 2000.

[29] D. Bertsimas and J. N. Tsitsiklis, Introduction to Linear Optimization. Belmont, MA: Athena Scientific, 1997.
[30] CVX Research Inc., "CVX: Matlab software for disciplined convex programming, version 2.0 (beta)," http://cvxr.com/cvx, Sep. 2012.

[31] MOSEK, http://www.mosek.com/, 2012.

[32] MISO Market Data, [Online]. Available: https://www. midwestiso.org/MarketsOperations/RealTimeMarketData/Pages/ DayAheadWindForecast.aspx.

[33] C. Wu, H. Mohsenian-Rad, J. Huang, and Y. Wang, "Demand side management for wind power integration in microgrid using dynamic potential game theory," in Proc. of 2011 IEEE GLOBECOM Workshops, Houston, TX, Dec. 5-9, 2011.

[34] Federal Energy Regulatory Commission, "MISO daily report," Feb. 29, 2012, [Online]. Available: http://www.ferc.gov/market-oversight/mktelectric/midwest/miso-rto-dly-rpt.pdf.

[35] U. Faigle, W. Kern, and G. Still, Algorithmic Principles of Mathematical Programming. Norwell, MA: Kluwer Academic Publishers, 2002. 